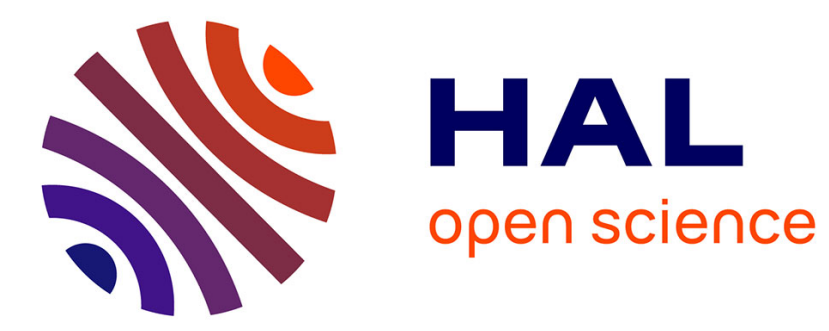

\title{
Lyapunov-Krasovskii characterization of the input-to-state stability for switching retarded systems
}

Ihab I. Haidar, Pierdomenico Pepe

\section{To cite this version:}

Ihab I. Haidar, Pierdomenico Pepe. Lyapunov-Krasovskii characterization of the input-to-state stability for switching retarded systems. 2021. hal-03233502

\section{HAL Id: hal-03233502 \\ https://hal.science/hal-03233502}

Preprint submitted on 24 May 2021

HAL is a multi-disciplinary open access archive for the deposit and dissemination of scientific research documents, whether they are published or not. The documents may come from teaching and research institutions in France or abroad, or from public or private research centers.
L'archive ouverte pluridisciplinaire HAL, est destinée au dépôt et à la diffusion de documents scientifiques de niveau recherche, publiés ou non, émanant des établissements d'enseignement et de recherche français ou étrangers, des laboratoires publics ou privés. 


\title{
Lyapunov-Krasovskii characterization of the input-to-state stability for switching retarded systems *
}

\author{
Ihab Haidar ${ }^{\dagger}$ and Pierdomenico Pepe ‡ $^{\ddagger}$
}

May 24, 2021

\begin{abstract}
In this paper we characterize various stability notions of nonlinear switching retarded systems by the existence of a common Lyapunov-Krasovskii functional with suitable conditions. We consider a general class of Lebesgue measurable switching signals. We provide an equivalence property showing that uniform input-to-state stability can be equivalently studied through the class of piecewise-constant inputs and piecewise-constant switching signals. Thanks to this equivalence property, we rely on what it is developed in the literature to provide direct and converse theorems for uniform input-to-state, asymptotic and exponential stability. Based on these results, we give a first order approximation theorem for nonlinear switching retarded systems. A link between the exponential stability of an unforced switching retarded system and the input-to-state stability property, in the case of measurable switching signals, is obtained. Examples showing the applicability of our results are also given.
\end{abstract}

Keywords: Input-to-state stability; Converse theorems; Lyapunov-Krasovskii functionals; retarded functional differential equations; switching systems.

\section{Introduction}

Many complex systems encountered in practice result from switching phenomenon between different individual subsystems [3]. Mathematically, a switched system can be defined by an indexed family of dynamical subsystems and a rule that orchestrates the switching between them. The problem of stability and stabilizability of such class of systems has motivated an interesting branch in the literature of control theory (see, e.g., $[1,2,22,23,28,42,43,46]$ and references therein). It is well known that the existence of a Lyapunov function which is independent on the switching index with some uniform dissipation property along the trajectories of each individual subsystem, called common Lyapunov function, consists of a sufficient condition for various stability notions. The necessity question about the existence of a common Lyapunov function for switching systems which are uniformly stable has attracted much attention in the literature of control theory. Converse Lyapunov theorems for the global asymptotic stability of switching systems have been developed in $[3,26,44]$ for finite-dimensional systems, in [10, 15, 31] for infinite-dimensional systems, and in [11, 12, 13] for switching retarded systems. These theorems are also a key for characterizing another important stability notion called input-to-state stability (ISS), which is introduced by E. Sontag in [39]. Indeed, the well known approach introduced in [40] for ordinary differential equations, characterizing the ISS by some robust global asymptotic stability, allows to use the converse theorems developed for global asymptotic stability in order to prove the existence of a Lyapunov function for systems which are ISS. This approach is further used for switching ordinary differential systems [25], as well as for retarded and neutral functional differential systems without switch $[19,38]$.

\footnotetext{
${ }^{*}$ This work is supported by a public grant overseen by the Research and Valorization Service (SRV) of ENSEA.

${ }^{\dagger}$ Quartz EA 7393, ENSEA, Cergy-Pontoise, France, ihab.haidar@ensea.fr

${ }^{\ddagger}$ Department of Information Engineering, Computer Science, and Mathematics, University of L’Aquila, 67100 L’Aquila, Italy pierdomenico.pepe@univaq.it.
} 
In this paper we consider a general class of switching retarded systems with measurable switching signal. Inspired by what is developed in [11], we start by proving that the ISS property (as well as the asymptotic and exponential stability) of switching retarded systems can be equivalently studied through the class of piecewise-constant inputs and piecewise-constant switching signals. More precisely, we show that a switching retarded system is uniformly ISS (uniformity is meant here with respect to the switching signal) if and only if this property holds for all piecewise-constant inputs and piecewise-constant switching signals. At the best of the authors' knowledge, this equivalence property has not been explored in the literature of input-to-state stability, not even for finite-dimensional non-switching systems. Different important points arise from this equivalence property. For example, an important point concerns the problem of the absolute continuity of Lyapunov-Krasovskii functionals. In fact, when dealing with a retarded system, the map describing the evolution of the state is simply continuous with respect to time (see, e.g., [14, Lemma 2.1]). Thus a continuous, or even Lipschitz on bounded sets, Lyapunov-Krasovskii functional evaluated on the solutions of such a system will be in general continuous and not absolutely continuous with respect to time. By consequence, when we deal with a retarded equation which holds almost everywhere (this is, for example, the case of systems with Lebesgue measurable inputs), the nonpositivity, almost everywhere, of the upper righthand Dini derivative of a Lyapunov-Krasovskii functional is not sufficient to conclude about its monotonicity. Different solutions have been proposed in the literature in order to overcome this problem (see, e.g., [17, 35]). For example, in [35], one proves that this problem can be overcome by restricting the class of initial states to continuously differentiable ones; this does not yield any loss of generality because, as it is shown in the same paper, the ISS property holds with continuous initial states if and only if it holds with continuously differentiable ones. Here, thanks to our equivalence property, this problem can be overcome differently by restricting the class of inputs and switching signals to the class of piecewise-constant ones. Indeed, in this case, the nonpositivity of the upper right-hand Dini derivative of a Lyapunov-Krasovskii functional evaluated on the solutions of a switching retarded system will hold everywhere instead of almost everywhere permitting to conclude about the monotonicity question (see [9]). By the virtue of this equivalence property, we further generalize what is recently developed in [13] concerning uniform asymptotic and exponential stability of switching retarded systems with piecewise-continuous switching signals to the case of measurable switching ones. Also, based on the same approach used in [19, 38, 40], we provide, thanks to the converse theorems developed in [13], a characterization of the ISS property of switching retarded systems through the existence of a Lyapunov-Krasovskii functional with suitable conditions.

Thanks to these theorems, a link between the exponential stability of an unforced switching retarded system and the input-to-state stability, in the case of Lebesgue measurable switching signals, is given, thus extending what is recently developed in [13] in the case of piecewise-constant switching signals. We also extend the very well known first order approximation theorem (see, e.g. [20, 21]) to nonlinear switching retarded systems. More precisely, we show that a nonlinear switched retarded system is uniformly locally exponentially stable if and only if its linearized one is uniformly globally exponentially stable, provided that, for each mode, the nonlinear map describing the related dynamics is Fréchet differentiable at the origin. This theorem is very useful in practice. In fact, the local stability analysis of a nonlinear switching retarded system can be then reduced to the stability analysis of a switching linear one, for which many useful methods exist in the literature (see, e.g., $[27,45]$ ). The theorems developed in this paper are also applicable to nonlinear systems with uncertain Lebesgue measurable time-delays. These results further generalize previous ones in the literature concerning retarded systems with time-varying delays assumed piecewise continuous (see [17]).

The paper is organized as follows. Section 2 presents the notation, definitions and assumptions in use. The statements of our main results are presented in Section 3. The link between exponential stability and input-to-state stability, and the first order approximation theorem are given in Sections 4 and 5, respectively. Two examples describing the applicability of our results are shown in Section 6. All the proofs are postponed to the appendix.

\section{Switching retarded systems}

In this section we list the notation, definitions, and the main assumptions in use. 


\section{$2.1 \quad$ Notation}

Throughout the paper, we adopt the following notation: $\mathbb{R}$ denotes the set of real numbers, $\mathbb{R}_{+}$the set of non-negative real numbers, and $\overline{\mathbb{R}}$ the extended real line. By $\left(\mathbb{R}^{n},\|\cdot\|\right)$ we denote the $n$-dimensional Euclidean space, where $n$ is a positive integer and $\|\cdot\|$ is the Euclidean norm. Given $r>0, B(0, r)$ denotes the closed ball of $\left(\mathbb{R}^{n},\|\cdot\|\right)$ of center 0 and radius $r$. The length of an interval $I \subset \mathbb{R}$ is denoted by $|I|$. By $\mathbb{1}_{I}$ we denote the indicator function of a nonempty subset $I$ of $\mathbb{R}$.

Given $\Delta>0, \mathcal{C}=\left(\mathcal{C}\left([-\Delta, 0], \mathbb{R}^{n}\right),\|\cdot\|_{\infty}\right)$ denotes the Banach space of continuous functions mapping $[-\Delta, 0]$ into $\mathbb{R}^{n}$, where $\|\cdot\|_{\infty}$ is the norm of uniform convergence. For a function $x:[-\Delta, b) \rightarrow \mathbb{R}^{n}$, with $0<b \leq+\infty$, for $t \in[0, b), x_{t}:[-\Delta, 0] \rightarrow \mathbb{R}^{n}$ denotes the history function defined by $x_{t}(\theta)=x(t+\theta)$, $-\Delta \leq \theta \leq 0$. For a positive real $H$ and given $\phi \in \mathcal{C}, \mathcal{C}_{H}(\phi)$ denotes the subset $\left\{\psi \in \mathcal{C}:\|\phi-\psi\|_{\infty} \leq H\right\}$. We simply denote $\mathcal{C}_{H}(0)$ by $\mathcal{C}_{H}$.

A measurable function $u: \mathbb{R}_{+} \rightarrow \mathbb{R}^{m}, m$ positive integer, is said to be essentially bounded if $\operatorname{ess} \sup _{t \geq 0}|u(t)|<$ $+\infty$. We use the symbol $\|\cdot\|_{\infty}$ to indicate the essential supremum norm of an essentially bounded function. For given times $0 \leq t_{1}<t_{2}, u_{\left[t_{1}, t_{2}\right)}: \mathbb{R}_{+} \rightarrow \mathbb{R}^{m}$ indicates the function given by $u_{\left[t_{1}, t_{2}\right)}=u(t) \mathbb{1}_{\left[t_{1}, t_{2}\right)}(t)$ for $t \geq 0$. A function $u: \mathbb{R}_{+} \rightarrow \mathbb{R}^{m}$ is said to be locally essentially bounded if, for any $t>0, u_{[0, t)}$ is essentially bounded.

A function $\alpha: \mathbb{R}_{+} \rightarrow \mathbb{R}_{+}$is said to be of class $\mathcal{K}$ if it is continuous, strictly increasing and $\gamma(0)=0$; it is said to be of class $\mathcal{K}_{\infty}$ if it is of class $\mathcal{K}$ and unbounded. A continuous function $\beta: \mathbb{R}_{+} \times \mathbb{R}_{+} \rightarrow \mathbb{R}_{+}$is said to be of class $\mathcal{K} \mathcal{L}$ if $\beta(\cdot, t)$ is of class $\mathcal{K}$ for each $t \geq 0$ and, for each $s \geq 0, \beta(s, \cdot)$ is nonincreasing and converges to zero as $t$ tends to $+\infty$.

With the symbol $\|\cdot\|_{a}$ we indicate any semi-norm in $\mathcal{C}\left([-\Delta, 0], \mathbb{R}^{n}\right)$ such that, for some positive constants $\underline{\gamma_{a}}$ and $\overline{\gamma_{a}}$, the following inequalities hold:

$$
\underline{\gamma_{a}}|\phi(0)| \leq\|\phi\|_{a} \leq \overline{\gamma_{a}}\|\phi\|_{\infty}, \quad \forall \phi \in \mathcal{C}\left([-\Delta, 0], \mathbb{R}^{n}\right) .
$$

The symbol $\circ$ denotes composition of functions and the symbol $\otimes$ denotes the Kronecker product of matrices. By $\operatorname{co}(E)$ we denote the convex hull of a nonempty subset $E$ of $\mathbb{R}^{n}$.

\subsection{Definitions and assumptions}

Let us consider the switching control system described by the following retarded functional differential equation

$$
\Sigma: \begin{array}{ll}
\dot{x}(t)=f_{\sigma(t)}\left(x_{t}, u(t)\right), & \text { a.e. } t \geq 0 \\
x(\theta)=x_{0}(\theta), & \theta \in[-\Delta, 0]
\end{array}
$$

where: $x(t) \in \mathbb{R}^{n} ; n$ is a positive integer; $\Delta$ is a positive real (the maximum involved time delay); $x_{0} \in \mathcal{C}$ is the initial state; the function $\sigma: \mathbb{R}_{+} \rightarrow \mathrm{S}$ is the switching signal; $\mathrm{S}$ is a nonempty set; $u: \mathbb{R}_{+} \rightarrow \mathbb{R}^{m}, m$ positive integer, is a Lebesgue measurable locally essentially bounded input signal.

We introduce the following two assumptions:

Assumption 1. For each $s \in \mathrm{S}, f_{s}(0,0)=0$. Moreover, $f_{s}(\cdot, \cdot)$ is uniformly (with respect to s $\in \mathrm{S}$ ) Lipschitz on bounded subsets of $\mathcal{C} \times \mathbb{R}^{m}$, i.e., for any $H>0$ there exists $L_{H}>0$ such that for every $\varphi, \psi \in \mathcal{C}_{H}$ and $u, v \in B(0, H)$, the following inequality holds

$$
\left|f_{s}(\varphi, u)-f_{s}(\psi, v)\right| \leq L_{H}\left(\|\varphi-\psi\|_{\infty}+|u-v|\right), \quad \forall s \in \mathrm{S} .
$$

We denote by $\mathcal{U}$ the set of Lebesgue measurable locally essentially bounded inputs from $\mathbb{R}_{+}$to $\mathbb{R}^{m}$ and by $\mathcal{U}^{\mathrm{PC}}$ the subset of right-continuous piecewise-constant ones. We denote also by $\mathcal{S}$ the set of measurable signals $\sigma: \mathbb{R}_{+} \rightarrow \mathrm{S}$ and by $\mathcal{S}^{\mathrm{PC}}$ the subset of right-continuous piecewise-constant ones.

Assumption 2. For each $\phi \in \mathcal{C}, \sigma \in \mathcal{S}$ and $u \in \mathcal{U}$, the function $t \mapsto f_{\sigma(t)}(\phi, u(t)), t \in \mathbb{R}_{+}$, is Lebesgue measurable. 
Under Assumption 1 and Assumption 2, the existence and uniqueness of a solution for system $\Sigma$ as well as its continuous dependence on the initial state is guaranteed by the theory of systems described by retarded functional differential equations (see, e.g., $[14,21]$ ). This can be reformulated by the following lemma.

Lemma 3. For any $\phi \in \mathcal{C}, u \in \mathcal{U}$ and $\sigma \in \mathcal{S}$, there exists, uniquely, a locally absolutely continuous solution $x(t, \phi, u, \sigma)$ of $\Sigma$ in a maximal time interval $[0, b)$, with $0<b \leq+\infty$. If $b<+\infty$, then the solution is unbounded in $[0, b)$. Moreover, for any $\varepsilon>0$, for any $c \in(0, b)$, there exists $\delta>0$ such that, for any $\psi \in \mathcal{C}_{\delta}(\phi)$, the solution $x(t, \psi, u, \sigma)$ exists in $[0, c]$ and, furthermore, the following inequality holds

$$
|x(t, \phi, u, \sigma)-x(t, \psi, u, \sigma)| \leq \varepsilon, \quad \forall t \in[0, c] .
$$

We give in the following the different definitions of uniform exponential, asymptotic and input-to-state stability of system $\Sigma$. The uniformity here is with respect to the switching signals $\sigma$.

Definition 4. We say that system $\Sigma$, with $u(t) \equiv 0$, is M-0-GAS (PC-0-GAS, respectively), if there exist a function $\beta \in \mathcal{K} \mathcal{L}$ such that, for any $x_{0} \in \mathcal{C}$ and $\sigma \in \mathcal{S}$ ( $\mathcal{S}^{\mathrm{PC}}$, respectively), the corresponding solution exists in $\mathbb{R}_{+}$and, furthermore, satisfies the inequality

$$
\left|x\left(t, x_{0}, \sigma\right)\right| \leq \beta\left(\left\|x_{0}\right\|_{\infty}, t\right), \quad \forall t \geq 0 .
$$

Definition 5. We say that system $\Sigma$, with $u(t) \equiv 0$, is M-0-GES (PC-0-GES, respectively), if there exist positive reals $M$ and $\lambda$ such that, for any $x_{0} \in \mathcal{C}$ and $\sigma \in \mathcal{S}$ (S ${ }^{\mathrm{PC}}$, respectively), the corresponding solution exists in $\mathbb{R}_{+}$and, furthermore, satisfies the inequality

$$
\left|x\left(t, x_{0}, \sigma\right)\right| \leq M e^{-\lambda t}\left\|x_{0}\right\|_{\infty}, \quad \forall t \geq 0 .
$$

Definition 6. We say that system $\Sigma$, with $u(t) \equiv 0$, is M-0-LES (PC-0-LES, respectively), if there exist positive reals $M, \lambda$ and $H$ such that, for any $x_{0} \in \mathcal{C}_{H}$ and $\sigma \in \mathcal{S}$ (S ${ }^{\mathrm{PC}}$, respectively), the corresponding solution exists in $\mathbb{R}_{+}$and, furthermore, satisfies the inequality

$$
\left|x\left(t, x_{0}, \sigma\right)\right| \leq M e^{-\lambda t}\left\|x_{0}\right\|_{\infty}, \quad \forall t \geq 0 .
$$

Definition 7. We say that system $\Sigma$ is M-ISS (PC-ISS, respectively), if there exist a function $\beta \in \mathcal{K} \mathcal{L}$ and a class $\mathcal{K}$ function $\gamma$ such that, for any $x_{0} \in \mathcal{C}, u \in \mathcal{U}\left(\mathcal{U}^{\mathrm{PC}}\right.$, respectively) and $\sigma \in \mathcal{S}\left(\mathcal{S}^{\mathrm{PC}}\right.$, respectively), the corresponding solution exists in $\mathbb{R}_{+}$and, furthermore, satisfies the inequality

$$
\left|x\left(t, x_{0}, u, \sigma\right)\right| \leq \beta\left(\left\|x_{0}\right\|_{\infty}, t\right)+\gamma\left(\left\|u_{[0, t)}\right\|_{\infty}\right), \quad \forall t \geq 0 .
$$

Let us also recall the following definition about Driver's form derivative of a continuous functional $V$ : $\mathcal{C} \rightarrow \mathbb{R}_{+}$. This definition is a variation of the one given in $[5,34,36]$ for retarded functional differential equations without switching.

Definition 8. For a continuous functional $V: \mathcal{C} \rightarrow \mathbb{R}_{+}$, its Driver's form derivative, $D^{+} V: \mathcal{C} \times \mathbb{R}^{m} \rightarrow \overline{\mathbb{R}}$, is defined, for the switching system $\Sigma$, for $\phi \in \mathcal{C}$ and $u \in \mathbb{R}^{m}$, as follows,

$$
D^{+} V(\phi, u)=\sup _{s \in \mathrm{S}} \limsup _{h \rightarrow 0^{+}} \frac{V\left(\phi_{h, u}^{\Sigma, s}\right)-V(\phi)}{h},
$$

where $\phi_{h, u}^{\Sigma, s} \in \mathcal{C}$ is defined, for $h \in[0, \Delta)$ and $\theta \in[-\Delta, 0]$, as follows

$$
\phi_{h, u}^{\Sigma, s}(\theta)= \begin{cases}\phi(\theta+h), & \theta \in[-\Delta,-h) \\ \phi(0)+(\theta+h) f_{s}(\phi, u), & \theta \in[-h, 0] .\end{cases}
$$




\section{Main results}

\subsection{Input-to-state stability characterization theorems}

In this section we give two different characterizations of the input-to-state stability property of system $\Sigma$. The first one is based on the following theorem which shows that this property can be equivalently studied through the class of piecewise-constant inputs and piecewise-constant switching signals.

Theorem 9. System $\Sigma$ is M-ISS if and only if it is PC-ISS.

At the best of the authors' knowledge, the result stated by Theorem 9 has not been explored in the literature related to the property of input-to-state stability, not even in the framework of non-switching finite-dimensional systems. The proof of Theorem 9 is given in Appendix A.

The second characterization of the input-to-state stability property of system $\Sigma$ is given through the existence of a common Lyapunov-Krasovskii functional. This theorem is in the spirit of the converse LyapunovKrasovskii theorems developed in $[18,19,38]$ for systems described by retarded and neutral functional differential equations.

Theorem 10. System $\Sigma$ is M-ISS if and only if there exist a functional $V: \mathcal{C} \rightarrow \mathbb{R}_{+}$, Lipschitz on bounded subsets of $\mathcal{C}$, functions $\alpha_{1}, \alpha_{2}, \alpha_{3} \in \mathcal{K}_{\infty}$, and $\alpha_{4} \in \mathcal{K}$ such that the following inequalities hold:

(i) $\alpha_{1}(|\phi(0)|) \leq V(\phi) \leq \alpha_{2}\left(\|\phi\|_{a}\right), \quad \forall \varphi \in \mathcal{C}$,

(ii) $D^{+} V(\phi, u) \leq-\alpha_{3}\left(\|\phi\|_{a}\right)+\alpha_{4}(|u|), \quad \forall \varphi \in \mathcal{C}, \forall u \in \mathbb{R}^{m}$.

The proof of Theorem 10 is based on the approach introduced in [41] in the case of non-switching finitedimensional systems. The proof is given in Apendix B.

\subsection{Asymptotic and exponential stability characterization theorems}

The following theorems provide necessary and sufficient conditions for the asymptotic and exponential stability properties of system $\Sigma$. The result is given through the existence of a common Lyapunov-Krasovskii functional. These theorems are in the spirit of the converse Lyapunov-Krasovskii theorems developed in $[17,18,19,37]$ for systems described by nonlinear retarded and neutral functional differential equations, and in [13] for switching retarded functional differential equations with piecewise-constant switching signals.

Theorem 11. System $\Sigma$ is M-0-GAS if and only if there exist a functional $V: \mathcal{C} \rightarrow \mathbb{R}_{+}$, Lipschitz on bounded subsets of $\mathcal{C}$, and functions $\alpha_{1}, \alpha_{2} \in \mathcal{K}_{\infty}, \alpha_{3} \in \mathcal{K}$ such that the following inequalities hold for any $\phi \in \mathcal{C}$

(i) $\alpha_{1}(|\phi(0)|) \leq V(\phi) \leq \alpha_{2}\left(\|\phi\|_{\infty}\right)$

(ii) $D^{+} V(\phi) \leq-\alpha_{3}(|\phi(0)|)$.

Theorem 12. System $\Sigma$ is M-0-GES if and only if there exist a functional $V: \mathcal{C} \rightarrow \mathbb{R}_{+}$, Lipschitz on bounded subsets of $\mathcal{C}$, and positive reals $\alpha_{1}, \alpha_{2}, \alpha_{3}$ and $p$, such that the following inequalities hold for any $\phi \in \mathcal{C}$

(i) $\alpha_{1}\|\phi\|_{\infty}^{p} \leq V(\phi) \leq \alpha_{2}\|\phi\|_{\infty}^{p}$,

(ii) $D^{+} V(\phi) \leq-\alpha_{3}\|\phi\|_{\infty}^{p}$.

The following theorem gives necessary and sufficient conditions for the M-0-LES property of system $\Sigma$.

Theorem 13. System $\Sigma$ is M-0-LES if and only if there exist positive reals $H, p, \alpha_{1}, \alpha_{2}, \alpha_{3}$ and Lipschitz functional $V: \mathcal{C}_{H} \rightarrow \mathbb{R}_{+}$, such that the following inequalities hold for any $\phi \in \mathcal{C}_{H}$

(i) $\alpha_{1}\|\phi\|_{\infty}^{p} \leq V(\phi) \leq \alpha_{2}\|\phi\|_{\infty}^{p}$,

(ii) $D^{+} V(\phi) \leq-\alpha_{3}\|\phi\|_{\infty}^{p}$.

The proofs of Theorem 11, Theorem 12 and Theorem 13 are given in Appendix C. 


\section{A link between exponential stability and input-to-state stability}

We have the following theorem (inspired by $[13,47]$ ).

Theorem 14. Let system $\Sigma$ be PC-0-GES. If there exist positive reals $L$ and $l$ and a nonnegative real $p<1$ such that:

1) $\forall \phi, \psi \in \mathcal{C}, \forall u \in \mathbb{R}^{m}, \forall s \in \mathrm{S}$, the following inequality holds

$$
\left|f_{s}(\phi, u)-f_{s}(\psi, u)\right| \leq L\|\phi-\psi\|_{\infty} ;
$$

2) $\forall \phi \in \mathcal{C}, \forall u \in \mathbb{R}^{m}, \forall s \in \mathrm{S}$, the following inequality holds

$$
\left|f_{s}(\phi, u)-f_{s}(\phi, 0)\right| \leq l \max \left\{\|\phi\|_{\infty}^{p}, 1\right\}|u| ;
$$

then system $\Sigma$ is M-ISS.

The proof of Theorem 14 is given in Appendix D. Notice that the hypothesis of this theorem concerns piecewise-constant switching signals, and the result concerns Lebesgue measurable switching signals. This theorem is therefore an extension of the result provided in [13].

\section{$5 \quad$ Stability by first order approximation}

While Theorem 13 characterizes the M-0-LES property of the nonlinear switching system $\Sigma$ through the existence of a common Lyapunov-Krasovskii functional, the following theorem uses its first-order approximation. In addition to Assumption 1 and 2, this theorem requires that the maps $f_{s}$ are Fréchet differentiable at 0 , for each $s \in \mathrm{S}$.

Definition 15. A functional $f: \mathcal{C} \rightarrow \mathbb{R}^{n}$ is said to be Fréchet differentiable at $\phi \in \mathcal{C}$ if there exists a bounded linear operator $L(\phi): \mathcal{C} \rightarrow \mathbb{R}^{n}$ (the Fréchet derivative at $\phi$ ) such that

$$
\lim _{\psi \rightarrow 0, \psi \neq 0} \frac{|f(\phi+\psi)-f(\phi)-L(\phi) \psi|}{\|\psi\|_{\infty}}=0 .
$$

Theorem 16. Let, for $s \in \mathrm{S}$, the map $f_{s}$ be Fréchet differentiable at 0 , and let $L_{s}$ be the Fréchet derivative at 0 . Suppose that $L_{s}$ is uniformly (with respect to $s \in \mathrm{S}$ ) bounded, i.e., there exists $m>0$, such that

$$
\left|L_{s} \phi\right| \leq m\|\phi\|_{\infty}, \quad \forall \phi \in \mathcal{C}, \forall s \in \mathrm{S} .
$$

Then system $\Sigma$ is M-0-LES if and only if the linear switching system

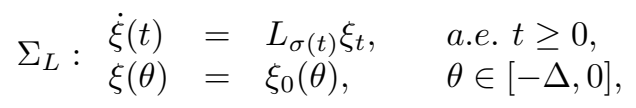

with $\xi_{0} \in \mathcal{C}$, is PC-0-GES.

The proof of Theorem 16 is obtained by means of the equivalence between PC-0-GES and M-0-GES properties, together with the developed converse Lyapunov-Krasovskii results. Theorem 16 is one of the many significant cases which converse Lyapunov-Krasovskii theorems are useful for (see Section 6). The proof is given in Appendix E. 


\section{Illustrative examples}

Example 17. Consider the following nonlinear uncertain time-varying retarded functional differential equation

$$
\begin{aligned}
& \dot{x}(t)=f\left(\tau(t), x_{t}, u(t)\right), \quad \text { a.e. } t \geq 0, \\
& x(\theta)=x_{0}(\theta), \quad \theta \in[-\Delta, 0] \text {, }
\end{aligned}
$$

where: $x(t) \in \mathbb{R}^{n} ; \tau: \mathbb{R} \rightarrow \mathrm{S}$ are Lebesgue measurable time-delay functions; $\mathrm{S} \subset[0, \Delta]^{D}, D$ positive integer, $\Delta$ positive real (maximum involved time delay); $u: \mathbb{R}_{+} \rightarrow \mathbb{R}^{m}, m$ positive integer, is a Lebesgue measurable locally essentially bounded input; $f: \mathrm{S} \times \mathcal{C} \times \mathbb{R}^{m} \rightarrow \mathbb{R}^{n}$ is a continuous function such that $f(s, 0,0)=0$ and $f(s, \cdot, \cdot)$ is Lipschitz on bounded subsets of $\mathcal{C} \times \mathbb{R}^{m}$ uniformly with respect to $s \in \mathrm{S}$.

For each $s \in \mathrm{S}$, let, with a slight abuse of notation, $f_{s}: \mathcal{C} \times \mathbb{R}^{m} \rightarrow \mathbb{R}^{n}$ be the function which is defined in $\mathcal{C} \times \mathbb{R}^{m}$ as

$$
f_{s}(\phi, v)=f(s, \phi, v), \quad \phi \in \mathcal{C}, v \in \mathbb{R}^{m} .
$$

So, system (3) can be equivalently rewritten as system $\Sigma$ with $\sigma \equiv \tau$. The existence and uniqueness of the solution of system (3) is guaranteed by Assumption 1 and Assumption 2. Thanks to this equivalent switching representation and thanks to Theorem 10, we have that system (3) is M-ISS if and only if there exist a functional $V: \mathcal{C} \rightarrow \mathbb{R}_{+}$, Lipschitz on bounded subsets of $\mathcal{C}$, and functions $\alpha_{1}, \alpha_{2}, \alpha_{3} \in \mathcal{K}_{\infty}$, and $\alpha_{4} \in \mathcal{K}$, such that inequalities (i) and (ii) of Theorem 10 are satisfied. This result further generalizes previous ones in the literature concerning retarded systems with time-varying delays assumed piecewise continuous (see [17]).

Example 18. Consider a system described by the following equation

$$
\begin{array}{ll}
\dot{x}(t)=A x(t)+B x\left(t-\tau_{0}\right)+C\left(\tau_{1}(t)\right)\left(x\left(t-\tau_{2}(t)\right) \otimes x\left(t-\tau_{3}(t)\right)\right), & \text { a.e. } t \geq 0, \\
x(\theta)=x_{0}(\theta), & \theta \in[-\Delta, 0],
\end{array}
$$

where: $x(t) \in \mathbb{R}^{n} ; \tau_{0} \in[0, \Delta] ; \tau_{1}, \tau_{2}, \tau_{3}: \mathbb{R}_{+} \rightarrow[0, \Delta]$ are measurable uncertain functions; $A$ and $B$ are $n \times n$ real matrices; the matrix function $C:[0, \Delta] \rightarrow \mathbb{R}^{n \times n^{2}}$ is continuous. Let $\mathrm{S}=[0, \Delta]^{3}$ and let $\tau: \mathbb{R} \rightarrow \mathrm{S}$ be the measurable function, which is defined, for each $t \in \mathbb{R}_{+}$, by $\tau(t)=\left(\tau_{1}(t), \tau_{2}(t), \tau_{3}(t)\right)$. For each $s=\left(s_{1}, s_{2}, s_{3}\right) \in \mathrm{S}$, let the function $f_{s}: \mathcal{C} \rightarrow \mathbb{R}^{n}$ be defined, for $\phi \in \mathcal{C}$, as

$$
f_{s}(\phi)=A \phi(0)+B \phi\left(-\tau_{0}\right)+C\left(s_{1}\right)\left(\phi\left(-s_{2}\right) \otimes \phi\left(-s_{3}\right)\right) .
$$

System (4) can be equivalently written as system $\Sigma$. Furthermore, Assumptions 1 and 2 are satisfied. Indeed, Assumption 1 is a straightforward consequence of the fact that the matrix function $C(\cdot)$ is continuous. Concerning Assumption 2, for each $\phi \in \mathcal{C}$, the function $g: \mathbb{R}_{+} \rightarrow \mathbb{R}^{n}$ defined, for $t \in \mathbb{R}_{+}$, as $g(t)=f_{\tau(t)}(\phi)$ is clearly measurable. By Theorem 16, system (4) is M-0-LES if and only if the linear, time-invariant system described by

$$
\begin{array}{lll}
\dot{\xi}(t)=A \xi(t)+B \xi\left(t-\tau_{0}\right), & & t \geq 0, \\
\xi(\theta)=x_{0}(\theta), & & \theta \in[-\Delta, 0],
\end{array}
$$

is PC-0-GES. Thus, in order to study the M-0-LES property of system (4), we can refer to the linear system (5). Many methods, such as the ones based on LMIs, are available in the literature which permit to examine the exponential stability of systems like (5) (see, e.g., [6, 7, 8, 32], and references therein). If, in the case of system (5), these LMIs are verified, then the M-0-LES property of system (4) follows from Theorem 16. As well, eigenvalues based approaches (see [29]), or CTCR methods (see [33]) can be used for establishing the exponential stability of (5), from which the M-0-LES property of system (4) follows from Theorem 16.

\section{Acknowledgements}

The authors are grateful to ENSEA and DISIM-Univaq for warm hospitality during the preparation of this work. 


\section{A Proof of Theorem 9}

The proof is inspired from [11]. The proof of $\Sigma$ is M-ISS implies that it is PC-ISS is obvious. Suppose now that $\Sigma$ is PC-ISS and let $\beta$ and $\gamma$ be the related $\mathcal{K} \mathcal{L}$ and $\mathcal{K}$ functions. Let $\phi \in \mathcal{C}, u \in \mathcal{U}$ and $\sigma \in \mathcal{S}$. Let $x(t, \phi, u, \sigma)$ be the corresponding solution in a maximal interval $[0, b), 0<b \leq+\infty$. We are going to prove, using a contradiction reasoning, that

$$
|x(t, \phi, u, \sigma)| \leq \beta\left(\|\phi\|_{\infty}, t\right)+\gamma\left(\left\|u_{[0, t)}\right\|_{\infty}\right), \quad \forall t \in[0, b) .
$$

Recall that (see Lemma 3) inequality (6) implies $b=+\infty$. Let us suppose the existence of $0<\ell<b$ such that

$$
|x(\ell, \phi, u, \sigma)|>\beta\left(\|\phi\|_{\infty}, \ell\right)+\gamma\left(\left\|u_{[0, \ell)}\right\|_{\infty}\right) .
$$

For $k \geq 1$, consider the bounded function $I_{j} \ni t \mapsto f_{\sigma(t)}\left(x_{j \frac{\ell}{k}}, u(t)\right)$, and $I_{j}=\left[(j-1) \frac{\ell}{k}, j \frac{\ell}{k}\right), 1 \leq j \leq k$. Let $\mathcal{I}=\left\{t \in[0, \ell]:|u(t)|>\left\|u_{[0, \ell]}\right\|_{\infty}\right\}$ and let $\bar{u} \in \mathcal{U}$ be the function defined as $\bar{u}(t)=u(t) \mathbb{1}_{[0, \ell] \backslash \mathcal{I}}(t)$. We remark that $\frac{k}{\ell} \int_{I_{j}} f_{\sigma(\tau)}\left(x_{j \frac{\ell}{k}}, u(\tau)\right) d \tau=\frac{k}{\ell} \int_{I_{j}} f_{\sigma(\tau)}\left(x_{j \frac{\ell}{k}}, \bar{u}(\tau)\right) d \tau$ is in the closure of the convex hull of (see, e.g., [4, Chapter 2, Corollary 8])

$$
E=\left\{f_{s}\left(x_{j \frac{\ell}{k}}, v\right): s \in \mathrm{S}, v \in \bar{u}\left(I_{j}\right)\right\} \subset \mathbb{R}^{n},
$$

where $\bar{u}\left(I_{j}\right)=\left\{\bar{u}(t), t \in I_{j}\right\}$. Thus, for each $1 \leq j \leq k$ there exist an element $\mathcal{E}_{j} \in \operatorname{co}(E)$ such that

$$
\left|\frac{k}{\ell} \int_{I_{j}} f_{\sigma(\tau)}\left(x_{j \frac{\ell}{k}}, u(\tau)\right) d \tau-\mathcal{E}_{j}\right|<\frac{1}{k} .
$$

Carathéodory's Theorem (see, e.g., [16, Chapter 3, Theorem 1.3.6]) implies that $\mathcal{E}_{j}$ is a convex combination of $n+1$ points of $E$, i.e.,

$$
\mathcal{E}_{j}=\sum_{i=1}^{n+1} \alpha_{i j} f_{s_{i j}}\left(x_{j \frac{\ell}{k}}, u_{i j}\right),
$$

where $s_{i j} \in \mathrm{S}, u_{i j} \in \bar{u}\left(I_{j}\right), \alpha_{i j}$ is nonnegative and $\sum_{i=1}^{n+1} \alpha_{i j}=1$. Substitute $\mathcal{E}_{j}$ by its value in (8), we get the following inequality

$$
\left|\frac{k}{\ell} \int_{I_{j}} f_{\sigma(\tau)}\left(x_{j \frac{\ell}{k}}, u(\tau)\right) d \tau-\sum_{i=1}^{n+1} \alpha_{i j} f_{s_{i j}}\left(x_{j \frac{\ell}{k}}, u_{i j}\right)\right|<\frac{1}{k} .
$$

Let $\sigma_{k}:[0, \ell] \rightarrow \mathrm{S}$ be the piecewise-constant signal defined as

$$
\sigma_{k}(t)=s_{i j} \quad \forall t \in I_{i j},
$$

and $u_{k}:[0, \ell] \rightarrow \mathbb{R}^{m}$ be the piecewise-constant input defined as

$$
u_{k}(t)=u_{i j} \quad \forall t \in I_{i j}
$$

where the (disjoint) intervals $I_{i j}$ are such that $\bigcup_{i=1}^{n+1} I_{i j}=I_{j}$ and $\left|I_{i j}\right|=\alpha_{i j} \frac{\ell}{k}$. We associate with $\left(\sigma_{k}, u_{k}\right)$ the following system

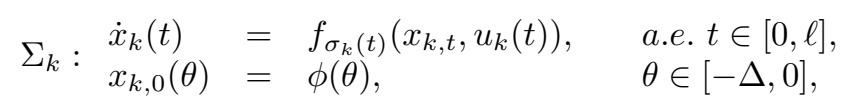

where $x_{k, t}:[-\Delta, 0] \rightarrow \mathbb{R}^{n}$ is defined by $x_{k, t}(\theta)=x_{k}(t+\theta),-\Delta \leq \theta \leq 0$.

Claim 19. $\forall \varepsilon>0, \exists k_{0} \geq 1$ such that $\forall k \geq k_{0}$, we have $\sup _{t \in[0, \ell]}\left\|x_{k, t}-x_{t}\right\|_{\infty}<\varepsilon$. 
Let us assume that the claim is true. Knowing that $\Sigma$ is PC-ISS, it follows that the solution of $\Sigma_{k}$ satisfies the following inequality

$$
\left|x_{k}\left(t, \phi, u_{k}, \sigma_{k}\right)\right| \leq \beta\left(\|\phi\|_{\infty}, t\right)+\gamma\left(\left\|u_{k[0, t)}\right\|_{\infty}\right), \quad \forall t \in[0, \ell] .
$$

From (7), it follows the existence of an $\varepsilon>0$ such that

$$
|x(\ell, \phi, u, \sigma)| \geq \varepsilon+\beta\left(\|\phi\|_{\infty}, \ell\right)+\gamma\left(\left\|u_{[0, \ell)}\right\|_{\infty}\right) .
$$

For this $\varepsilon$, thanks to the claim, there exits $k_{0}$ such that, for every $k \geq k_{0}$, we have

$$
\left|x_{k}\left(\ell, \phi, u_{k}, \sigma_{k}\right)-x(\ell, \phi, u, \sigma)\right|<\frac{\varepsilon}{2} .
$$

It follows, from (11) and (12), that

$$
\begin{aligned}
|x(\ell, \phi, u, \sigma)| & \leq\left|x_{k}\left(\ell, \phi, u_{k}, \sigma_{k}\right)\right|+\frac{\varepsilon}{2} \leq \beta\left(\|\phi\|_{\infty}, \ell\right)+\gamma\left(\left\|u_{k[0, \ell)}\right\|_{\infty}\right)+\frac{\varepsilon}{2} \\
& \leq \beta\left(\|\phi\|_{\infty}, \ell\right)+\gamma\left(\left\|u_{[0, \ell)}\right\|_{\infty}\right)+\frac{\varepsilon}{2}<|x(\ell, \phi, u, \sigma)|-\frac{\varepsilon}{2} .
\end{aligned}
$$

Hence the contradiction arises. It follows that

$$
|x(t, \phi, u, \sigma)| \leq \beta\left(\|\phi\|_{\infty}, t\right)+\gamma\left(\left\|u_{[0, t)}\right\|_{\infty}\right), \quad \forall t \in[0, b) .
$$

Inequality (13) holds for any $\phi \in \mathcal{C}, \sigma \in \mathcal{S}$, and any $u \in \mathcal{U}$. From inequality (13) and Lemma 3, it follows that $b=+\infty$ and $\Sigma$ is M-ISS.

Proof of the claim: Let, for $k \geq 1, \Gamma_{k}:[0, \ell] \rightarrow \mathbb{R}^{n}$ be the function defined as

$$
\Gamma_{k}(t)=\int_{0}^{t}\left(f_{\sigma(\tau)}\left(x_{\tau}(\phi, u, \sigma), u(\tau)\right)-f_{\sigma_{k}(\tau)}\left(x_{\tau}(\phi, u, \sigma), u_{k}(\tau)\right)\right) d \tau, \quad \forall t \in[0, \ell],
$$

and $y_{k}:[-\Delta, \ell] \rightarrow \mathbb{R}^{n}$ be the function defined as

$$
y_{k}(t)=x(t, \phi, u, \sigma)-x_{k}\left(t, \phi, u_{k}, \sigma_{k}\right), \quad \forall t \in[-\Delta, \ell] .
$$

We have, for $t \in[0, \ell]$,

$$
y_{k}(t)=\int_{0}^{t} f_{\sigma(\tau)}\left(x_{\tau}(\phi, u, \sigma), u(\tau)\right) d \tau-\int_{0}^{t} f_{\sigma_{k}(\tau)}\left(x_{k, \tau}\left(\phi, u_{k}, \sigma_{k}\right), u_{k}(\tau)\right) d \tau .
$$

By adding and subtracting $\int_{0}^{t} f_{\sigma_{k}(\tau)}\left(x_{\tau}(\phi, u, \sigma), u_{k}(\tau)\right) d \tau$ in the expression of $y_{k}(t)$, we get the following equalities/inequalities

$$
\begin{aligned}
\left|y_{k}(t)\right| & =\left|\int_{0}^{t} f_{\sigma(\tau)}\left(x_{\tau}(\phi, u, \sigma), u(\tau)\right) d \tau-\int_{0}^{t} f_{\sigma_{k}(\tau)}\left(x_{k, \tau}\left(\phi, u_{k}, \sigma_{k}\right), u_{k}(\tau)\right) d \tau\right| \\
& \leq \int_{0}^{t}\left|f_{\sigma_{k}(\tau)}\left(x_{\tau}(\phi, u, \sigma), u_{k}(\tau)\right)-f_{\sigma_{k}(\tau)}\left(x_{k, \tau}\left(\phi, u_{k}, \sigma_{k}\right), u_{k}(\tau)\right)\right| d \tau+\left|\Gamma_{k}(t)\right| \\
& \leq L_{H} \int_{0}^{t}\left\|x_{\tau}(\phi, u, \sigma)-x_{k, \tau}\left(\phi, u_{k}, \sigma_{k}\right)\right\|_{\infty} d \tau+\sup _{\tau \in[0, \ell]}\left|\Gamma_{k}(\tau)\right| \\
& =L_{H} \int_{0}^{t}\left\|y_{k, \tau}\right\|_{\infty} d \tau+\sup _{\tau \in[0, \ell]}\left|\Gamma_{k}(\tau)\right|
\end{aligned}
$$

where

$$
H=\max \left\{\sup _{\tau \in[-\Delta, \ell]}|x(\tau, \phi, u, \sigma)|, \sup _{\tau \in[-\Delta, \ell]}\left|x_{k}\left(\tau, \phi, u_{k}, \sigma_{k}\right)\right|\right\} .
$$


From (15), we have

$$
\begin{aligned}
\left\|y_{k, t}\right\|_{\infty} & =\sup _{\theta \in[-\Delta, 0]}\left|y_{k}(t+\theta)\right| \leq \sup _{\theta \in[-\Delta, 0], t+\theta \geq 0} L_{H} \int_{0}^{t+\theta}\left\|y_{k, \tau}\right\|_{\infty} d \tau+\sup _{\tau \in[0, \ell]}\left|\Gamma_{k}(\tau)\right|, \\
& \leq L_{H} \int_{0}^{t}\left\|y_{k, \tau}\right\|_{\infty} d \tau+\sup _{\tau \in[0, \ell]}\left|\Gamma_{k}(\tau)\right| .
\end{aligned}
$$

By Gronwall's lemma, the following inequality holds

$$
\sup _{t \in[0, \ell]}\left\|y_{k, t}\right\|_{\infty} \leq e^{L_{H} \ell} \sup _{t \in[0, \ell]}\left|\Gamma_{k}(t)\right| .
$$

We are going to show that $\Gamma_{k}$ converges uniformly to zero on the interval $[0, \ell]$. Observe that, for $t \in[0, \ell]$, we have

$$
\Gamma_{k}(t)=\sum_{j=1}^{k} \int_{I_{j} \cap[0, t]}\left(f_{\sigma(\tau)}\left(x_{\tau}(\phi, u, \sigma), u(\tau)\right)-f_{\sigma_{k}(\tau)}\left(x_{\tau}(\phi, u, \sigma), u_{k}(\tau)\right)\right) d \tau .
$$

Then, by adding and subtracting

$$
\sum_{j=1}^{k} \int_{I_{j} \cap[0, t]}\left(f_{\sigma_{k}(\tau)}\left(x_{j \frac{\ell}{k}}(\phi, u, \sigma), u_{k}(\tau)\right)-f_{\sigma(\tau)}\left(x_{j \frac{\ell}{k}}(\phi, u, \sigma), u(\tau)\right)\right) d \tau
$$

in the expression of $\Gamma_{k}$, the following equality/inequalities hold

$$
\begin{aligned}
\Gamma_{k}(t)= & \sum_{j=1}^{k} \int_{I_{j} \cap[0, t]}\left(f_{\sigma(\tau)}\left(x_{\tau}(\phi, u, \sigma), u(\tau)\right)-f_{\sigma(\tau)}\left(x_{j \frac{\ell}{k}}(\phi, u, \sigma), u(\tau)\right)\right) d \tau \\
& +\sum_{j=1}^{k} \int_{I_{j} \cap[0, t]}\left(f_{\sigma_{k}(\tau)}\left(x_{j \frac{\ell}{k}}(\phi, u, \sigma), u_{k}(\tau)\right)-f_{\sigma_{k}(\tau)}\left(x_{\tau}(\phi, u, \sigma), u_{k}(\tau)\right)\right) d \tau \\
& -\sum_{j=1}^{k} \int_{I_{j} \cap[0, t]}\left(f_{\sigma_{k}(\tau)}\left(x_{j \frac{\ell}{k}}(\phi, u, \sigma), u_{k}(\tau)\right)-f_{\sigma(\tau)}\left(x_{j \frac{\ell}{k}}(\phi, u, \sigma), u(\tau)\right)\right) d \tau \\
\leq & 2 L_{H} \sum_{j=1}^{k} \int_{I_{j} \cap[0, t]}\left\|x_{\tau}(\phi, u, \sigma)-x_{j \frac{\ell}{k}}(\phi, u, \sigma)\right\|_{\infty} d \tau-\sum_{j=1}^{k} K_{j},
\end{aligned}
$$

where, for $j=1, \cdots, k$, the constant $K_{j}$ is given by

$$
K_{j}=\int_{I_{j} \cap[0, t]}\left(f_{\sigma_{k}(\tau)}\left(x_{j \frac{\ell}{k}}(\phi, u, \sigma), u_{k}(\tau)\right)-f_{\sigma(\tau)}\left(x_{j \frac{\ell}{k}}(\phi, u, \sigma), u(\tau)\right)\right) d \tau .
$$

We distinguish three different cases:

(i) if $I_{j} \cap[0, t]=I_{j}$, then by definition of $f_{\sigma_{k}}$ and using inequality (9), we have

$$
\begin{aligned}
\left|K_{j}\right| & =\left|\int_{I_{j}} f_{\sigma_{k}(\tau)}\left(x_{j \frac{\ell}{k}}(\phi, u, \sigma), u_{k}(\tau)\right) d \tau-\int_{I_{j}} f_{\sigma(\tau)}\left(x_{j \frac{\ell}{k}}(\phi, u, \sigma), u(\tau)\right) d \tau\right| \\
& =\left|\sum_{i=1}^{n+1} \int_{I_{i j}} f_{s_{i j}}\left(x_{j \frac{\ell}{k}}(\phi, u, \sigma), u_{i j}\right) d \tau-\int_{I_{j}} f_{\sigma(\tau)}\left(x_{j \frac{\ell}{k}}(\phi, u, \sigma), u(\tau)\right) d \tau\right| \\
& =\frac{\ell}{k}\left|\sum_{i=1}^{n+1} \alpha_{i j} f_{s_{i j}}\left(x_{j \frac{\ell}{k}}(\phi, u, \sigma), u_{i j}\right)-\frac{k}{\ell} \int_{I_{j}} f_{\sigma(\tau)}\left(x_{j \frac{\ell}{k}}(\phi, u, \sigma), u(\tau)\right) d \tau\right| \\
& <\frac{\ell}{k^{2}}
\end{aligned}
$$


(ii) if $I_{j} \cap[0, t]=\left[(j-1) \frac{\ell}{k}, t\right]$, then

$$
\begin{aligned}
\left|K_{j}\right| & \leq \int_{\left[(j-1) \frac{\ell}{k}, t\right]}\left|f_{\sigma_{k}(\tau)}\left(x_{j \frac{\ell}{k}}(\phi, u, \sigma), u_{k}(\tau)\right)-f_{\sigma(\tau)}\left(x_{j \frac{\ell}{k}}(\phi, u, \sigma), u(\tau)\right)\right| d \tau \\
& \leq \int_{I_{j}}\left|f_{\sigma_{k}(\tau)}\left(x_{j \frac{\ell}{k}}(\phi, u, \sigma), u_{k}(\tau)\right)\right| d \tau+\int_{I_{j}}\left|f_{\sigma(\tau)}\left(x_{j \frac{\ell}{k}}(\phi, u, \sigma), u(\tau)\right)\right| d \tau \\
& \leq 2 L_{H} \int_{I_{j}}\left\|x_{j \frac{\ell}{k}}(\phi, u, \sigma)\right\|_{\infty} d \tau+\int_{I_{j}} L_{H}\left|u_{k}(\tau)\right| d \tau+\int_{I_{j}} L_{H}|u(\tau)| d \tau \\
& \leq 2 L_{H} \frac{\ell}{k}\left\|x_{j \frac{\ell}{k}}(\phi, u, \sigma)\right\|_{\infty}+2 \frac{\ell}{k} L_{H}\left\|u_{[0, \ell]}\right\|_{\infty} ;
\end{aligned}
$$

(iii) if $I_{j} \cap[0, t]=\emptyset$, then $K_{j}=0$.

For $\epsilon>0$ there exists $k_{1}$ such that if $k \geq k_{1}$ then $\sum_{j=1}^{k}\left|K_{j}\right|<\frac{\epsilon}{2 e^{L_{H} \ell}}$. Furthermore, Since $x(\cdot)$ is uniformly continuous on $[-\Delta, \ell]$, we deduce the existence of $k_{2}$ such that if $k \geq k_{2}$ then $\left\|x_{\tau}(\phi, u, \sigma)-x_{j \frac{\ell}{k}}(\phi, u, \sigma)\right\|_{\infty}<$ $\frac{\epsilon}{4 L_{H} \ell e^{L_{H} \ell}}$ for every $\tau \in I_{j}$ and every $j=1, \ldots, k$. As a consequence, if $k \geq \max \left\{k_{1}, k_{2}\right\}$ we have, from (17),

$$
\left|\Gamma_{k}(t)\right|<\frac{\epsilon}{4 L_{H} \ell e^{L_{H} \ell}} 2 L_{H} \sum_{j=1}^{k}\left|I_{j}\right|+\frac{\epsilon}{2 e^{L_{H} \ell}}=\frac{\epsilon}{L_{H} \ell} .
$$

Note that this is true for every $t \in[0, \ell]$, we conclude that

$$
\sup _{t \in[0, \ell]}\left|\Gamma_{k}(t)\right|<\frac{\epsilon}{L_{H} \ell} .
$$

Equations (16) together with (19) conclude the proof of the claim. The proof of the theorem is complete.

\section{B Proof of Theorem 10}

The proof is analogous to the proof given in [24] for finite dimensional systems. However, as mentioned in the introduction, since we deal with a retarded system, the map describing the evolution of the state is simply continuous with respect to time. Thus a Lyapunov-Krasovskii functional evaluated on the solutions of such a system will be in general continuous and not absolutely continuous with respect to time. By consequence, we cannot directly use the standard comparison lemma [24, Lemma 4.4] in the proof of the sufficiency part of Theorem 10. Instead, exploiting Theorem 9, one can use the following comparison lemma from [30].

Lemma 20. [30, Lemma 1] For each continuous and positive definite function $\alpha$, there exists a class $\mathcal{K} \mathcal{L}$ function $\beta_{\alpha}$ with the following property: if, for $T>0$ (or $\left.T=+\infty\right), y:[0, T) \rightarrow \mathbb{R}_{+}$is a continuous non-negative function which satisfies the inequality

$$
D^{+} y(t) \leq-\alpha(y(t)), \quad \forall t \in[0, T),
$$

where $D^{+} y$ denotes the upper-right Dini derivative of $y$, with $y(0)=y_{0} \in \mathbb{R}_{+}$, then it holds that

$$
y(t) \leq \beta_{\alpha}\left(y_{0}, t\right), \quad \forall t \in[0, T) .
$$

\section{B.1 Proof of the sufficiency part}

Thanks to Theorem 9, it is sufficient to consider the case when the switching signal $\sigma$ and the input $u$ are piecewise-constant. For this, let $x_{0} \in \mathcal{C}, u \in \mathcal{U}^{\mathrm{PC}}$, and $\sigma \in \mathcal{S}^{\mathrm{PC}}$. Let $x\left(t, x_{0}, u, \sigma\right)$ be the corresponding 
solution in a maximal interval $[0, b), 0<b \leq+\infty$. Let $w:[0, b) \rightarrow \mathbb{R}_{+}$be the function which is defined in $[0, b)$ as

$$
w(t)=V\left(x_{t}\left(x_{0}, u, \sigma\right)\right), \quad \forall t \in[0, b) .
$$

Knowing that $u$ and $\sigma$ are piecewise-constant, then, by [5, 34], we have, for all $t \in[0, b)$,

$$
\begin{aligned}
D^{+} w(t) & =\limsup _{h \rightarrow 0^{+}} \frac{V\left(x_{t+h}\left(x_{0}, u, \sigma\right)\right)-V\left(x_{t}\left(x_{0}, u, \sigma\right)\right)}{h} \\
& =\limsup _{h \rightarrow 0^{+}} \frac{V\left(\left(x_{t}\left(x_{0}, u, \sigma\right)\right)_{h, u(t)}^{\Sigma, \sigma(t)}\right)-V\left(x_{t}\left(x_{0}, u, \sigma\right)\right)}{h} \\
& \leq \sup _{s \in \mathrm{S}} \limsup _{h \rightarrow 0^{+}} \frac{V\left(\left(x_{t}\left(x_{0}, u, \sigma\right)\right)_{h, u(t)}^{\Sigma, s}\right)-V\left(x_{t}\left(x_{0}, u, \sigma\right)\right)}{h} \\
& =D^{+} V\left(x_{t}\left(x_{0}, u, \sigma\right), u(t)\right) .
\end{aligned}
$$

By inequality (ii), it follows from (22) that

$$
D^{+} w(t) \leq-\alpha_{3}\left(\left\|x_{t}\left(x_{0}, u, \sigma\right)\right\|_{a}\right)+\alpha_{4}(|u(t)|), \quad \forall t \in[0, b) .
$$

Let the input $u(t)$ be such that $\sup _{t \geq 0}|u(t)|=v$, for a suitable $v \geq 0$. By analogous reasoning as in [36], we can prove the existence of a constant $c \in(0, b]$ such that

$$
\begin{array}{ll}
D^{+} w(t) \leq-\alpha(w(t)), & \forall t \in[0, c), \\
\left|x\left(t, x_{0}, u, \sigma\right)\right| \leq \gamma(v), & \forall t \in[c, b),
\end{array}
$$

where $\alpha=\frac{1}{2} \alpha_{3} \circ \alpha_{2}^{-1}$ and $\gamma=\alpha_{2} \circ \alpha_{3}^{-1} \circ 2 \alpha_{4}$. Since $t \mapsto w(t)$ is continuous, from Lemma 20 it holds the existence of a class $\mathcal{K} \mathcal{L}$ function $\beta_{\alpha}$ such that

$$
|w(t)| \leq \beta_{\alpha}(w(0), t), \quad \forall t \in[0, c),
$$

from which it follows that

$$
\left|x\left(t, x_{0}, u, \sigma\right)\right| \leq \beta\left(\left\|x_{0}\right\|_{\infty}, t\right), \quad \forall t \in[0, c),
$$

with $\beta(r, t)=\alpha_{1}^{-1} \circ \beta_{\alpha}\left(\alpha_{2}\left(\overline{\gamma_{a}} r\right), t\right)$. By consequence, inequalities (24) and (25) lead to the following inequality

$$
\left|x\left(t, x_{0}, u, \sigma\right)\right| \leq \beta\left(\left\|x_{0}\right\|_{\infty}, t\right)+\gamma(v), \quad \forall t \in[0, b) .
$$

It follows, from Lemma 3 , that $b=+\infty$. By causality arguments, and given the arbitrarity of $\varphi \in \mathcal{C}, u \in \mathcal{U}^{\mathrm{PC}}$ and $\sigma \in \mathcal{S}^{\mathrm{PC}}$, the PC-ISS of system $\Sigma$ is proved.

\section{B.2 Proof of the necessity part}

Let us consider the system described by the following retarded functional differential equation

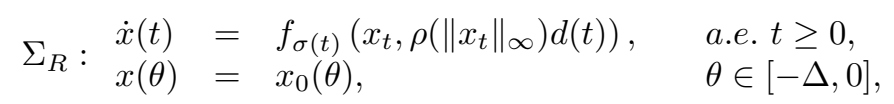

where $\rho$ is globally 1-Lipschitz of class $\mathcal{K}_{\infty}$ function, and $d \in \mathcal{M}_{B}$, where $\mathcal{M}_{B}$ is the set of Lebesgue measurable functions $g: \mathbb{R}_{+} \rightarrow \mathbb{R}^{m}$ satisfying ess $\sup _{\tau \geq 0}|g(\tau)| \leq 1$. Let us introduce the following definition:

Definition 21. We say that system $\Sigma_{R}$ is M-robustly 0 -GAS if there exists a class $\mathcal{K} \mathcal{L}$ function $\beta$ such that, for any initial state $x_{0} \in \mathcal{C}$, any $\sigma \in \mathcal{S}$ and any $d \in \mathcal{M}_{B}$, the corresponding solution, denoted by $x_{t}^{R}\left(x_{0}, d, \sigma\right)$, exists in $\mathbb{R}_{+}$and, furthermore satisfies the following inequality

$$
\left\|x_{t}^{R}\left(x_{0}, d, \sigma\right)\right\| \leq \beta\left(\left\|x_{0}\right\|_{\infty}, t\right), \quad \forall t \geq 0 .
$$


The proof of the necessity part follows the same methodology introduced in [41] for finite-dimensional systems and which was then extended in $[19,38]$ to encompass the class of retarded and neutral functional differential equations. This is given by the following three lemmas:

Lemma 22. If system $\Sigma$ is M-ISS, then there exists a suitable globally 1-Lipschitz of class $\mathcal{K}_{\infty}$ function $\rho$ such that the related system $\Sigma_{R}$ is M-robustly 0-GAS.

Proof. Knowing that we deal with 0-GAS property which is uniform with respect to $\sigma$ and $d$, the proof follows the same lines of the proof of [38, Lemma 17].

Before giving the second lemma, let us first recall the following Driver's form derivative of a LyapunovKrasovskii functional related to system $\Sigma_{R}$

$$
D^{+} V(\phi)=\sup _{s \in \mathrm{S}, d \in \mathcal{M}_{B}} \limsup _{h \rightarrow 0^{+}} \frac{V\left(\phi_{h, d}^{R, s}\right)-V(\phi)}{h},
$$

where, for $\varphi \in \mathcal{C}, h \in[0, \Delta)$, and $\theta \in[-\Delta, 0], \phi_{h, d}^{R, s} \in \mathcal{C}$ is defined as follows

$$
\phi_{h, d}^{R, s}(\theta)= \begin{cases}\phi(\theta+h), & \theta \in[-\Delta,-h) \\ \phi(0)+(\theta+h) f_{s}\left(\phi, \rho\left(\|\phi\|_{\infty}\right) d\right), & \theta \in[-h, 0] .\end{cases}
$$

We have the following lemma:

Lemma 23. Suppose that system $\Sigma_{R}$ is M-robustly 0-GAS. Then, there exist a functional $V: \mathcal{C} \rightarrow \mathbb{R}_{+}$, Lipschitz on bounded sets, and functions, $\alpha_{1}, \alpha_{2}, \alpha_{3}$ of class $\mathcal{K}_{\infty}$, such that, for any $\phi \in \mathcal{C}, \sigma \in \mathcal{S}$ and $d \in B_{\mathbb{R}^{m}}(0,1)$, the following inequalities hold:

(i) $\alpha_{1}\left(\|\phi\|_{\infty}\right) \leq V(\phi) \leq \alpha_{2}\left(\|\phi\|_{\infty}\right)$

(ii) $D^{+} V(\phi) \leq-\alpha_{3}\left(\|\phi\|_{\infty}\right)$.

Proof. The proof follows the same lines of the proof of the necessity part in [13, Theorem 1].

We have also the following lemma:

Lemma 24. [38, Lemma 21] Let $V: \mathcal{C} \rightarrow \mathbb{R}_{+}$, be a Lipschitz on bounded sets functional such that there exists functions, $\alpha_{1}, \alpha_{2}$ of class $\mathcal{K}_{\infty}$ such that the following inequalities hold:

$$
\alpha_{1}\left(\|\phi\|_{\infty}\right) \leq V(\phi) \leq \alpha_{2}\left(\|\phi\|_{\infty}\right), \quad \forall \phi \in \mathcal{C} .
$$

Then, the following conditions are equivalent:

(i) there exist a class $\mathcal{K}_{\infty}$ function $\gamma$ and a class $\mathcal{K}$ function $\rho$ such that, for every $\phi \in \mathcal{C}, u \in \mathbb{R}^{m}$ such that $\|\phi\|_{\infty} \geq \rho(|u|)$, the inequality holds,

$$
D^{+} V(\phi, u) \leq-\gamma\left(\|\phi\|_{\infty}\right)
$$

(ii) there exist a class $\mathcal{K}_{\infty}$ function $\alpha_{3}$ and a class $\mathcal{K}$ function $\alpha_{4}$ such that, for every $\phi \in \mathcal{C}, u \in \mathbb{R}^{m}$ the following inequality holds,

$$
D^{+} V(\phi, u) \leq-\alpha_{3}\left(\|\phi\|_{\infty}\right)+\alpha_{4}(|u|)
$$

The proof of the necessity part of Theorem 10 derives from the following results: if system $\Sigma$ is M-ISS then, from Lemma 22, there exists a 1-Lipschitz of class $\mathcal{K}_{\infty}$ function $\rho$ such that system $\Sigma_{R}$ is M-robustly 0GAS. Thus, from Lemma 23, there exist a functional $V: \mathcal{C} \rightarrow \mathbb{R}_{+}$, Lipschitz on bounded sets, and functions, $\alpha_{1}, \alpha_{2}, \omega_{3}$ of class $\mathcal{K}_{\infty}$, such that, for any $\phi \in \mathcal{C}, \sigma \in \mathcal{S}$ and $u \in \mathbb{R}^{m}$, satisfying $|u| \leq \rho\left(\|\phi\|_{\infty}\right)$, the following inequalities hold: 
(i) $\alpha_{1}\left(\|\phi\|_{\infty}\right) \leq V(\phi) \leq \alpha_{2}\left(\|\phi\|_{\infty}\right)$

(ii) $D^{+} V(\phi) \leq-\omega_{3}\left(\|\phi\|_{\infty}\right)$.

Finally, thanks to Lemma 24, the point (ii) is equivalent to the existence of a class $\mathcal{K}_{\infty}$ function $\alpha_{3}$ and a class $\mathcal{K}$ function $\alpha_{4}$ such that, for every $\phi \in \mathcal{C}, u \in \mathbb{R}^{m}$ the following inequality holds,

(iii) $D^{+} V(\phi, u) \leq-\alpha_{3}\left(\|\phi\|_{\infty}\right)+\alpha_{4}(|u|)$.

By consequence, if system $\Sigma$ is M-ISS then there exist a functional $V: \mathcal{C} \rightarrow \mathbb{R}_{+}$, Lipschitz on bounded sets, and functions $\alpha_{1}, \alpha_{2}, \alpha_{3}$ of class $\mathcal{K}_{\infty}$, and a class $\mathcal{K}$ function $\alpha_{4}$ such that, for any $\phi \in \mathcal{C}, \sigma \in \mathcal{S}$ and $u \in \mathbb{R}^{m}$, inequalities (i) and (iii) hold. Hence, the proof of the necessity part is complete.

\section{Proofs of Theorems 11, 12 and 13}

\section{C.1 Proof of Theorem 11}

The proof of Theorem 11 is a straightforward consequence of Theorem 9 together with the following theorem:

Theorem 25 ([13]). System $\Sigma$ is PC-0-GAS if and only if there exist a functional $V: \mathcal{C} \rightarrow \mathbb{R}_{+}$, Lipschitz on bounded subsets of $\mathcal{C}$, and functions $\alpha_{1}, \alpha_{2} \in \mathcal{K}_{\infty}, \alpha_{3} \in \mathcal{K}$ such that the following inequalities hold for any $\phi \in \mathcal{C}$

(i) $\alpha_{1}(|\phi(0)|) \leq V(\phi) \leq \alpha_{2}\left(\|\phi\|_{\infty}\right)$,

(ii) $D^{+} V(\phi) \leq-\alpha_{3}(|\phi(0)|)$.

\section{C.2 Proof of Theorem 12}

The proof of Theorem 12 is a straightforward consequence of the following theorem:

Theorem 26 ([13]). System $\Sigma$ is PC-0-GES if and only if there exist a functional $V: \mathcal{C} \rightarrow \mathbb{R}_{+}$, Lipschitz on bounded subsets of $\mathcal{C}$, and positive reals $\alpha_{1}, \alpha_{2}, \alpha_{3}$ and $p$, such that the following inequalities hold for any $\phi \in \mathcal{C}$

(i) $\alpha_{1}\|\phi\|_{\infty}^{p} \leq V(\phi) \leq \alpha_{2}\|\phi\|_{\infty}^{p}$,

(ii) $D^{+} V(\phi) \leq-\alpha_{3}\|\phi\|_{\infty}^{p}$.

together with the following lemma:

Lemma 27. System $\Sigma$ is M-0-GES if and only if it is PC-0-GES.

Proof. The proof follows the same lines of the proof of Theorem 9, and therefore is omitted.

\section{C.3 Proofs of Theorem 13}

The proof of Theorem 13 follows from the following two lemmas:

Lemma 28. System $\Sigma$ is M-0-LES if and only if it is PC-0-LES.

Proof. The proof follows the same lines of the proof of Theorem 9, and therefore is omitted.

Lemma 29. System $\Sigma$ is PC-0-LES if and only if there exist positive reals $H, p, \alpha_{1}, \alpha_{2}, \alpha_{3}$ and Lipschitz functional $V: \mathcal{C}_{H} \rightarrow \mathbb{R}_{+}$, such that the following inequalities hold for any $\phi \in \mathcal{C}_{H}$

(i) $\alpha_{1}\|\phi\|_{\infty}^{p} \leq V(\phi) \leq \alpha_{2}\|\phi\|_{\infty}^{p}$, 
(ii) $D^{+} V(\phi) \leq-\alpha_{3}\|\phi\|_{\infty}^{p}$.

Proof. We first prove the necessity part. Knowing that $\Sigma$ is PC-0-LES, then there exist positive reals $r, M$ and $\lambda$ such that the following inequality holds in $\mathcal{C}_{r}$

$$
\left|x\left(t, x_{0}, \sigma\right)\right| \leq M e^{-\lambda t}\left\|x_{0}\right\|_{\infty}, \quad \forall t \geq 0 .
$$

By taking $H=r / M$ and following the same lines of the proof of the necessity part of [13, Theorem 2], we get the proof. As far as the proof of the sufficiency part is concerned, let $\delta=\frac{1}{2}\left(\frac{\alpha_{1}}{\alpha_{2}}\right)^{\frac{1}{p}} H$. Let $x_{0} \in \mathcal{C}_{\delta}$ and let $\sigma \in \mathcal{S}^{\mathrm{PC}}$. Let $x\left(t, x_{0}, \sigma\right)$ be the corresponding solution in a maximal interval $[0, b)$. Let $w:[0, b) \rightarrow \mathbb{R}_{+}$ be the function which is defined in $[0, b)$ as

$$
w(t)=V\left(x_{t}\left(x_{0}, \sigma\right)\right), \quad t \in[0, b) .
$$

Claim 30. The solution $x\left(t, x_{0}, \sigma\right)$ stays in $\mathcal{C}_{H}$ for any $t \in[0, b)$.

Let us assume that the claim is true. We have, for all $t \in[0, b)$,

$$
\begin{aligned}
D^{+} w(t) & =\limsup _{h \rightarrow 0^{+}} \frac{w(t+h)-w(t)}{h}=\limsup _{h \rightarrow 0^{+}} \frac{V\left(x_{t+h}\left(x_{0}, \sigma\right)\right)-V\left(x_{t}\left(x_{0}, \sigma\right)\right)}{h} \\
& =\limsup _{h \rightarrow 0^{+}} \frac{V\left(\left(x_{t}\left(x_{0}, \sigma\right)\right)_{h}^{\Sigma, \sigma(t)}\right)-V\left(x_{t}\left(x_{0}, \sigma\right)\right)}{h} \\
& \leq \sup _{s \in \mathrm{S}} \limsup _{h \rightarrow 0^{+}} \frac{V\left(\left(x_{t}\left(x_{0}, \sigma\right)\right)_{h}^{\Sigma, s}\right)-V\left(x_{t}\left(x_{0}, \sigma\right)\right)}{h}=D^{+} V\left(x_{t}\left(x_{0}, \sigma\right)\right) .
\end{aligned}
$$

By inequalities (i) and (ii), it follows from (29) that

$$
D^{+} w(t) \leq-\alpha_{3}\left\|x_{t}\left(x_{0}, \sigma\right)\right\|_{\infty}^{p} \leq-\frac{\alpha_{3}}{\alpha_{2}} w(t), \quad \forall t \in[0, b) .
$$

Since $w$ is continuous, it follows (see [9]) that

$$
w(t) \leq e^{-\frac{\alpha_{3}}{\alpha_{2}} t} w(0), \quad \forall t \in[0, b) .
$$

Finally, from (31) and the inequalities (i), we obtain

$$
\left|x\left(t, x_{0}, \sigma\right)\right| \leq\left(\frac{\alpha_{2}}{\alpha_{1}}\right)^{\frac{1}{p}} e^{-\frac{\alpha_{3}}{p \alpha_{2}} t}\left\|x_{0}\right\|_{\infty}, \quad \forall t \in[0, b) .
$$

It follows, from Lemma 3 , that $b=+\infty$, and, given the arbitrarity of $x_{0} \in \mathcal{C}_{\delta}$ and $\sigma \in \mathcal{S}^{\mathrm{PC}}$, the PC-0-LES property of the system $\Sigma$ is proved, provided that the claim is true. Now, we prove the claim by contradiction. Assume there exists $t_{1} \in[0, b)$ such that $\left\|x_{t_{1}}\left(x_{0}, \sigma\right)\right\|_{\infty}<H, t \in\left[0, t_{1}\right)$, and $\left|x\left(t_{1}, x_{0}, \sigma\right)\right|=H$. Then, for $t \in\left[0, t_{1}\right]$, the inequalities (29)-(32) hold. Therefore, we obtain the following equalities/inequalities

$$
H=\left|x\left(t_{1}, x_{0}, \sigma\right)\right| \leq\left(\frac{\alpha_{2}}{\alpha_{1}}\right)^{\frac{1}{p}} e^{-\frac{\alpha_{3}}{p \alpha_{2}} t_{1}}\left\|x_{0}\right\|_{\infty} \leq\left(\frac{\alpha_{2}}{\alpha_{1}}\right)^{\frac{1}{p}} \delta=\frac{H}{2} .
$$

Therefore, the absurd inequality $H \leq \frac{H}{2}$ arises. By contradiction, the claim is proved. The proof of the sufficiency part of the lemma is complete. The proof of the lemma is complete. 


\section{Proofs of Theorem 14}

The proof of Theorem 14 follows directly from Theorem 9 together with the following theorem:

Theorem 31 ([13]). Let system $\Sigma$ be PC-0-GES. If there exist positive reals $L$ and $l$ and a nonnegative real $p<1$ such that:

1) $\forall \phi, \psi \in \mathcal{C}, \forall u \in \mathbb{R}^{m}, \forall s \in \mathrm{S}$, the following holds

$$
\left|f_{s}(\phi, u)-f_{s}(\psi, u)\right| \leq L\|\phi-\psi\|_{\infty} ;
$$

2) $\forall \phi \in \mathcal{C}, \forall u \in \mathbb{R}^{m}, \forall s \in \mathrm{S}$, the following holds

$$
\left|f_{s}(\phi, u)-f_{s}(\phi, 0)\right| \leq l \max \left\{\|\phi\|_{\infty}^{p}, 1\right\}|u| ;
$$

then system $\Sigma$ is PC-ISS.

\section{E Proofs of Theorem 16}

Lemma 32. Let the map $f_{s}$ be uniformly (with respect to $s \in \mathrm{S}$ ) globally Lipschitz. System $\Sigma$ is PC-0-GES if and only if there exist a functional $V: \mathcal{C} \rightarrow \mathbb{R}_{+}$, and positive reals $\alpha_{1}, \alpha_{2}, \alpha_{3}$ and $\alpha_{4}$, such that the following inequalities hold for any $\phi, \psi \in \mathcal{C}$

(i) $\alpha_{1}\|\phi\|_{\infty} \leq V(\phi) \leq \alpha_{2}\|\phi\|_{\infty}$,

(ii) $D^{+} V(\phi) \leq-\alpha_{3}\|\phi\|_{\infty}$,

(iii) $|V(\phi)-V(\psi)| \leq \alpha_{4}\|\phi-\psi\|_{\infty}$.

Proof. The proof follows the same lines of the proof of [13, Theorem 2], and therefore is omitted. The global Lipschitz property of the functional $V$ is proved by exploiting the uniform global Lipschitz property of $f_{s}$, $s \in \mathrm{S}$.

Lemma 33. Let, for $s \in \mathrm{S}$, the map $f_{s}$ be Fréchet differentiable at 0 , and let $L_{s}$ be its associated Fréchet derivative. Suppose that $L_{s}$ is uniformly (with respect to $s$ ) bounded. Then, system $\Sigma$ is PC-0-LES if and only if the linear switching system

$$
\Sigma_{L}: \begin{array}{rlrl}
\dot{\xi}(t) & =L_{\sigma(t)} \xi_{t}, & & \text { a.e. } t \geq 0, \\
\xi(\theta) & =\xi_{0}(\theta), & \theta \in[-\Delta, 0],
\end{array}
$$

with $\xi_{0} \in \mathcal{C}$, is PC-0-GES.

Proof. We prove first the sufficiency part. From Lemma 32, it follows that there exist a functional $V: \mathcal{C} \rightarrow$ $\mathbb{R}_{+}$, and positive reals $\alpha_{1}, \alpha_{2}, \alpha_{3}$ and $\alpha_{4}$, such that the inequalities $(i),(i i),($ iii $)$, in Lemma 32, hold with respect to system $\Sigma_{L}$. Now, we will show that $\Sigma$ is PC-0-LES by means of this functional $V$. So, let us consider the Driver's derivative of $V$ with respect to system $\Sigma$, i.e.,

$$
D^{+} V(\phi)=\sup _{s \in \mathrm{S}} \limsup _{h \rightarrow 0^{+}} \frac{V\left(\phi_{h}^{\Sigma, s}\right)-V(\phi)}{h} .
$$


Then, we have

$$
\begin{aligned}
D^{+} V(\phi) & =\sup _{s \in \mathrm{S}} \limsup _{h \rightarrow 0^{+}} \frac{V\left(\phi_{h}^{\Sigma, s}\right)+V\left(\phi_{h}^{\Sigma_{L}, s}\right)-V\left(\phi_{h}^{\Sigma_{L}, s}\right)-V(\phi)}{h} \\
& \leq \sup _{s \in \mathrm{S}}\left[\limsup _{h \rightarrow 0^{+}} \frac{V\left(\phi_{h}^{\Sigma, s}\right)-V\left(\phi_{h}^{\Sigma_{L}, s}\right)}{h}+\limsup _{h \rightarrow 0^{+}} \frac{V\left(\phi_{h}^{\Sigma_{L}, s}\right)-V(\phi)}{h}\right] \\
& \leq \sup _{s \in \mathrm{S}} \limsup _{h \rightarrow 0^{+}} \frac{\alpha_{4}\left\|\phi_{h}^{\Sigma, s}-\phi_{h}^{\Sigma_{L}, s}\right\|_{\infty}}{h}-\alpha_{3}\|\phi\|_{\infty} \\
& \leq \sup _{s \in \mathrm{S}} \limsup _{h \rightarrow 0^{+}} \frac{\alpha_{4} \sup _{\theta \in[-\Delta, 0]}\left|\phi_{h}^{\Sigma, s}(\theta)-\phi_{h}^{\Sigma_{L}, s}(\theta)\right|}{h}-\alpha_{3}\|\phi\|_{\infty} \\
& \leq \sup _{s \in \mathrm{S}} \limsup _{h \rightarrow 0^{+}} \frac{\alpha_{4} \sup _{\theta \in[-h, 0]}\left|\theta+h \| f_{s}(\phi)-L_{s} \phi\right|}{h}-\alpha_{3}\|\phi\|_{\infty} .
\end{aligned}
$$

Now, by using the definition of the Fréchet derivative of $f_{s}, s \in \mathrm{S}$, at 0 , let $\delta$ be a positive real such that if $\phi \in \mathcal{C}_{\delta}$ then the following inequality holds

$$
\left|f_{s}(\phi)-L_{s} \phi\right| \leq \frac{\alpha_{3}}{2 \alpha_{4}}\|\phi\|_{\infty}
$$

Therefore, for any $\phi \in \mathcal{C}_{\delta}$, we have

$$
D^{+} V(\phi) \leq \frac{\alpha_{3} \alpha_{4}}{2 \alpha_{4}}\|\phi\|_{\infty}-\alpha_{3}\|\phi\|_{\infty} \leq-\frac{\alpha_{3}}{2}\|\phi\|_{\infty}
$$

It follows, by Lemma 29, that system $\Sigma$ is PC-0-LES. The sufficiency part is proved. Now, we prove the necessity part. From Lemma 29, there exist positive reals $H, \alpha_{1}, \alpha_{2}, \alpha_{3}$ and Lipschitz functional $V: \mathcal{C}_{H} \rightarrow$ $\mathbb{R}_{+}$, such that the following inequalities hold

(i) $\alpha_{1}\|\phi\|_{\infty} \leq V(\phi) \leq \alpha_{2}\|\phi\|_{\infty}, \quad \forall \phi \in \mathcal{C}_{H}$,

(ii) $D^{+} V(\phi) \leq-\alpha_{3}\|\phi\|_{\infty}, \quad \forall \phi \in \mathcal{C}_{H}$,

(iii) $|V(\phi)-V(\psi)| \leq \alpha_{4}\|\phi-\psi\|_{\infty}, \quad \forall \phi, \psi \in \mathcal{C}_{H}$.

Let $\delta<H$ be a sufficiently small positive real such that, if $\phi \in \mathcal{C}_{\delta}$ then the following inequality holds

$$
\left|f_{s}(\phi)-L_{s} \phi\right| \leq \frac{\alpha_{3}}{2 \alpha_{4}}\|\phi\|_{\infty} .
$$

For sufficiently small $h$, for any $\phi \in \mathcal{C}_{\delta}$, and for any $s \in \mathrm{S}$, the following inequality holds

$$
\left|V\left(\phi_{h}^{\Sigma_{L}, s}\right)-V\left(\phi_{h}^{\Sigma, s}\right)\right| \leq \alpha_{4}\left\|\phi_{h}^{\Sigma_{L}, s}-\phi_{h}^{\Sigma, s}\right\|_{\infty}
$$

Let $\phi \in \mathcal{C}_{\delta}$. Calculating the Driver's derivative of $V$ with respect to system $\Sigma_{L}$ (by proceeding exactly as in (36) after interchanging $\Sigma$ by $\Sigma_{L}$ ), we obtain

$$
D^{+} V(\phi) \leq-\frac{\alpha_{3}}{2}\|\phi\|_{\infty}
$$

It follows, by Lemma 29, that system $\Sigma_{L}$ is PC-0-LES. From linearity it follows that system $\Sigma_{L}$ is PC-0-GES. The proof of the necessity part is complete. The proof of the lemma is complete.

The proof of Theorem 16 follows from Lemma 28 and Lemma 33. The proof of Theorem 16 is complete. 


\section{References}

[1] Andrei A. Agrachev and Daniel Liberzon. Lie-algebraic stability criteria for switched systems. SIAM Journal on Control and Optimization, 40(1):253-269, 2001.

[2] Ugo Boscain. Stability of planar switched systems: The linear single input case. SIAM Journal on Control and Optimization, 41(1):89-112, 2002.

[3] Wijesuriya P. Dayawansa and C. F. Martin. A converse Lyapunov theorem for a class of dynamical systems which undergo switching. IEEE Transactions on Automatic Control, 44(4):751-760, 1999.

[4] Joseph Diestel and John Jerry Uhl. Vector measures. American Mathematical Society, 1979.

[5] Rodney D. Driver. Existence and stability of solutions of a delay-differential system. Archive for Rational Mechanics and Analysis, 10(1):401-426, 1962.

[6] Boukas El-Kébir and Liu Zi-Kuan. Deterministic and stochastic time-delay systems. Birkaüser, Boston, 2002.

[7] Emilia Fridman. Introduction to time-delay systems: Analysis and control. Basel, Switzerland: Birkhaüser, 2014.

[8] Emilia Fridman and Jin Zhang. Averaging of linear systems with almost periodic coefficients: A timedelay approach. Automatica, 122:109287, 2020.

[9] John W. Hagood and Brian S. Thomson. Recovering a function from a Dini derivative. American Mathematical Monthly, 113(1):34-46, 2006.

[10] Ihab Haidar, Yacine Chitour, Paolo Mason, and Mario Sigalotti. Lyapunov characterization of uniform exponential stability for nonlinear infinite-dimensional systems. IEEE Transactions on Automatic Control, https://doi.org/10.1109/TAC.2021.3080526, 2021.

[11] Ihab Haidar, Paolo Mason, and Mario Sigalotti. Converse Lyapunov-Krasovskii theorems for uncertain retarded differential equations. Automatica, 62:263-273, 2015.

[12] Ihab Haidar, Paolo Mason, and Mario Sigalotti. Stability of interconnected uncertain delay systems: a converse Lyapunov approach. In G. Valmorbida, A. Seuret, I. Boussaada, and R. Sipahi, editors, Delays and Interconnections: Methodology, Algorithms and Applications, volume 10 of Advances in Delays and Dynamics. Springer, 2019.

[13] Ihab Haidar and Pierdomenico Pepe. Lyapunov-krasovskii characterizations of stability notions for switching retarded systems. IEEE Transactions on Automatic Control, 66(1):437-443, 2021.

[14] Jack K. Hale and Sjoerd M. Verduyn Lunel. Introduction to functional differential equations, volume 99. Springer-Verlag, 1993.

[15] Falk M. Hante and Mario Sigalotti. Converse Lyapunov theorems for switched systems in Banach and Hilbert spaces. SIAM Journal on Control and Optimization, 49(2):752-770, 2011.

[16] Jean-Baptiste Hiriart-Urruty and Claude Lemaréchal. Convex Analysis and Minimization Algorithms I, volume 305. Springer Berlin Heidelberg, 1993.

[17] Iasson Karafyllis. Lyapunov theorems for systems described by RFDEs. Nonlinear Analysis: Theory, Methods \& Applications, 64:590-617, 2006.

[18] Iasson Karafyllis, Pierdomenico Pepe, and Zhong P. Jiang. Global output stability for systems described by retarded functional differential equations: Lyapunov characterizations. European Journal of Control, 14:516-536, 2008. 
[19] Iasson Karafyllis, Pierdomenico Pepe, and Zhong P. Jiang. Input-to-output stability for systems described by retarded functional differential equations. European Journal of Control, 14:539-555, 2008.

[20] Hassan K. Khalil. Nonlinear systems. Prentice Hall, 2002.

[21] Vladimir B. Kolmanovskii and Anatoliy Myshkis. Introduction to the theory and applications of functional differential equations, volume. Dordrecht: Kluwer Academy, 1999.

[22] Daniel Liberzon. Systems \& Control: Foundations \& Applications. Birkhaüser Boston Inc., Boston, MA, 2003.

[23] Daniel Liberzon and A. Stephen Morse. Basic problems in stability and design of switched systems. IEEE Control Systems, 19(5):59-70, 1999.

[24] Yuandan Lin, Eduardo D. Sontag, and Yuan Wang. A smooth converse Lyapunov theorem for robust stability. SIAM Journal on Control and Optimization, 34(1):124-160, 1996.

[25] Jose L. Mancilla-Aguilar and R.A. García. On converse Lyapunov theorems for ISS and iISS switched nonlinear systems. Systems \& Control Letters, 42(1):47-53, 2001.

[26] Jose L. Mancilla-Aguilar and Ronaldo A. García. A converse Lyapunov theorem for nonlinear switched systems. Systems $\&$ Control Letters, 41(1):67-71, 2000.

[27] Paolo Mason, Ugo Boscain, and Yacine Chitour. Common polynomial Lyapunov functions for linear switched systems. SIAM Journal on Control and Optimization, 45(1):226-245, 2006.

[28] Frédéric Mazenc, Michael Malisoff, and Hitay Özbay. Stability and robustness analysis for switched systems with time-varying delays. SIAM Journal on Control and Optimization, 56(1):158-182, 2018.

[29] Wim Michiels and Silviu-Iulian Niculescu. Stability, Control, and Computation for Time-Delay Systems. Society for Industrial and Applied Mathematics, Philadelphia, PA, 2014.

[30] Andrii Mironchenko and Hiroshi Ito. Characterizations of integral input-to-state stability for bilinear systems in infinite dimensions. Mathematical Control \& Related Fields, 6(3):447-466, 2016.

[31] Andrii Mironchenko and Fabian Wirth. Non-coercive Lyapunov functions for infinite-dimensional systems. Journal of Differential Equations, 266(11):7038-7072, 2019.

[32] Silviu I. Niculescu. Delay effects on stability: A robust control approach, volume 269. Berlin: Springer, 2001.

[33] Nejat Olgac and Rifat Sipahi. An exact method for the stability analysis of time-delayed linear timeinvariant (LTI) systems. IEEE Transactions on Automatic Control, 47(5):793-797, 2002.

[34] Pierdomenico Pepe. On Liapunov-Krasovskii functionals under Carathéodory conditions. Automatica, 43(4):701-706, 2007.

[35] Pierdomenico Pepe. The problem of the absolute continuity for Lyapunov-Krasovskii functionals. IEEE Transactions on Automatic Control, 52:953-957, 2007.

[36] Pierdomenico Pepe and Zhong P. Jiang. A Lyapunov-Krasovskii methodology for ISS and iISS of time-delay systems. Systems \& Control Letters, 55(12):1006-1014, 2006.

[37] Pierdomenico Pepe and Iasson Karafyllis. Converse Lyapunov-Krasovskii theorems for systems described by neutral functional differential equations in Hale's form. International Journal of Control, 86(2):232-243, 2013. 
[38] Pierdomenico Pepe, Iasson Karafyllis, and Zhong P. Jiang. Lyapunov-Krasovskii characterization of the input-to-state stability for neutral systems in Hale's form. Systems \& Control Letters, 102:48-56, 2017.

[39] Eduardo D. Sontag. Smooth stabilization implies coprime factorization. IEEE Transactions on Automatic Control, 34(4):435-443, 1989.

[40] Eduardo D. Sontag. Comments on integral variants of ISS. Systems \& Control Letters, 34(1):93-100, 1998.

[41] Eduardo D. Sontag and Yuan Wang. On characterizations of the input-to-state stability property. Systems \& Control Letters, 24(5):351-359, 1995.

[42] Zhendong Sun and Shuzhi S. Ge. Stability theory of switched dynamical systems. Springer-Verlag, London, 2011.

[43] Yue-E Wang, Xi-Ming Sun, and Frédéric Mazenc. Stability of switched nonlinear systems with delay and disturbance. Automatica, 69:78 - 86, 2016.

[44] Fabian Wirth. A converse Lyapunov theorem for linear parameter-varying and linear switching systems. SIAM Journal on Control and Optimization, 44(1):210-239, 2005.

[45] Guangming Xie and Long Wang. Stability and stabilization of switched linear systems with state delay: Continuous-time case. In 16th International Symposium on Mathematical Theory of Networks and Systems, 2004.

[46] Peng Yan and Hitay Özbay. Stability analysis of switched time delay systems. SIAM Journal on Control and Optimization, 47(2):936-949, 2008.

[47] Nima Yeganefar, Pierdomenico Pepe, and Michel Dambrine. Input-to-state stability of time-delay systems: a link with exponential stability. IEEE Transactions on Automatic Control, 53(6):1526-1531, 2008. 